Roger E. Stoller ${ }^{1}$ and Lawrence R. Greenwood ${ }^{2}$

\title{
An Evaluation of Through-Thickness Changes in Primary Damage Production in Commercial Reactor Pressure Vessels
}

\begin{abstract}
Reference: Stoller, R. E. and Greenwood, L. R., "An Evaluation of Through-Thickness Changes in Primary Damage Production in Commercial Reactor Pressure Vessels," Effects of Radiation on Materials: 20th International Symposium, ASTM STP 1405, S. T. Rosinski, M. L. Grossbeck, T. R. Allen, and A. S. Kumar, Eds., American Society for Testing and Materials, West Conshohocken, PA, 2002.
\end{abstract}

\begin{abstract}
An extensive database of atomic displacement cascades in iron has been developed using the method of molecular dynamics (MD). More than 300 simulations have been completed at $100 \mathrm{~K}$ with energies between 0.1 and $100 \mathrm{keV}$. This encompasses nearly all energies relevant to fission reactor irradiation environments since a $100 \mathrm{keV} \mathrm{MD}$ cascade corresponds to the average iron cascade following a collision with a $5.1 \mathrm{MeV}$ neutron. Extensive statistical analysis of the database has determined representative average values for several primary damage parameters: the total number of surviving point defects, the fraction of the surviving point defects contained in clusters formed during cascade cooling, and a measure of the size distribution of the in-cascade point defect clusters. The cascade energy dependence of the MD-based primary damage parameters has been used to obtain spectrum-averaged defect production cross sections for typical fission reactor neutron energy spectra as a function of depth through the reactor pressure vessel. The attenuation of the spectrum-averaged cross sections for total point defect survival and the fraction of either interstitials or vacancies in clusters are quite similar to that for the NRT dpa. However, the cross sections derived to account for the energy dependence of the point defect cluster size distributions exhibit a potentially significant variation through the vessel. The production rate of large interstitial clusters decreases more rapidly than dpa whereas the production of large vacancy clusters is slower than dpa.
\end{abstract}

Keywords: damage attenuation, displacement cascades, ferritic steels, modeling, molecular dynamics, point defects, pressure vessels, radiation damage

\section{Introduction}

Current regulatory practice in the United States calls for radiation-induced reactor pressure vessel (RPV) embrittlement to be predicted on the basis of the neutron fluence above $1 \mathrm{MeV}[1,2]$. The correlation of embrittlement with the so-called fast fluence (i.e. neutron fluence above $1.0 \mathrm{MeV}$ ) is rationalized by the assumption that most atomic displacements are generated by the high energy portion of the spectrum, and is well

${ }^{1}$ Senior Research Staff, Oak Ridge National Laboratory, P. O. Box 2008, Oak Ridge, TN.

2 Lead Scientist, Pacific Northwest National Laboratory, P. O. Box 999, Richland, WA. 
supported by ongoing data analysis [3]. However, it is well known that neutrons of lower energy also contribute to embrittlement and the use of atomic displacements per atom (dpa) has been recommended [4,5] as an improved correlation parameter, particularly when differences in neutron energy spectrum must be accounted for. The change in neutron energy spectrum as a function of depth in the RPV is an important example of this situation. Because of this through-thickness variation in the neutron energy spectrum, Revision 2 of Regulatory Guide 1.99 (RG-1.99/2) specifies that either dpa or an empirical, dpa-based exponential be used to obtain an equivalent fast fluence for calculating Charpy shifts at locations within the vessel [1,2].

Differences in neutron energy spectra are manifested as differences in the energy spectra of the primary knockon atoms (PKA) produced in elastic collisions with these neutrons. Since low and high energy PKA can produce damage structures that are both qualitatively and quantitatively different from each other, a systematic investigation of the energy dependence of primary damage formation should provide some insight into how this damage may be attenuated through an RPV. The potential impact of differences in PKA energy spectra are amenable to investigation by displacement cascade simulations using the method of molecular dynamics (MD). MD simulations provide a detailed picture of the formation and evolution of displacement cascades, and recent advances in computing equipment permit the simulation of high energy displacement events involving several million atoms [6-10]. The results presented below encompass MD cascade simulation energies from near the displacement threshold to as high as $100 \mathrm{keV}$.

\section{RPV Irradiation Environment}

Representative neutron energy spectra were obtained from Ref. [11] for typical pressurized water [PWR] and boiling water reactors [BWR]. The analysis described below was carried out for both reactor types, but the results presented here will focus on the PWR. The conclusions reached for the BWR are essentially the same as for the PWR, with the primary difference being that the relative change between the inner and outer diameters of the BWR are reduced because the RPV thickness is $\sim 156 \mathrm{~mm}$ whereas the PWR RPV thickness is $\sim 218 \mathrm{~mm}$. Because of other design differences, the fast flux at the inner diameter of the RPV is lower for the BWR, $\sim 1.1 \times 10^{14} \mathrm{n} / \mathrm{m}^{2} / \mathrm{s}$, than for the PWR, $\sim 7.5 \times 10^{14} \mathrm{n} / \mathrm{m}^{2} / \mathrm{s}$. A previous analysis demonstrated that differences between the BWR and PWR neutron energy spectra were modest [12].

The neutron and PKA energy spectra for the PWR case are shown in Figs. 1 (a) and (b), respectively. To illustrate how the spectrum changes through the RPV, spectra are shown for three locations: $8 \mathrm{~mm}$ (the first RPV node from the transport calculations), and $1 / 4$ and 3/4 of the way through the RPV. The range of the MD cascade energies used in this study are included in Fig. 1(a), where the four cascade energies shown represent the average PKAs from the neutron energies indicated by the arrows. An MD energy of 0.1 $\mathrm{keV}$ is near the minimum required to yield stable displacements. Neutrons with energies below about $1 \mathrm{keV}$ produce atomic displacements primarily from the recoils generated by neutron capture $(n, \gamma)$ reactions. Such recoils have energies of a few hundred eV. PKA spectra calculated with the SPECTER code [13] for each of the neutron spectra in Fig. 

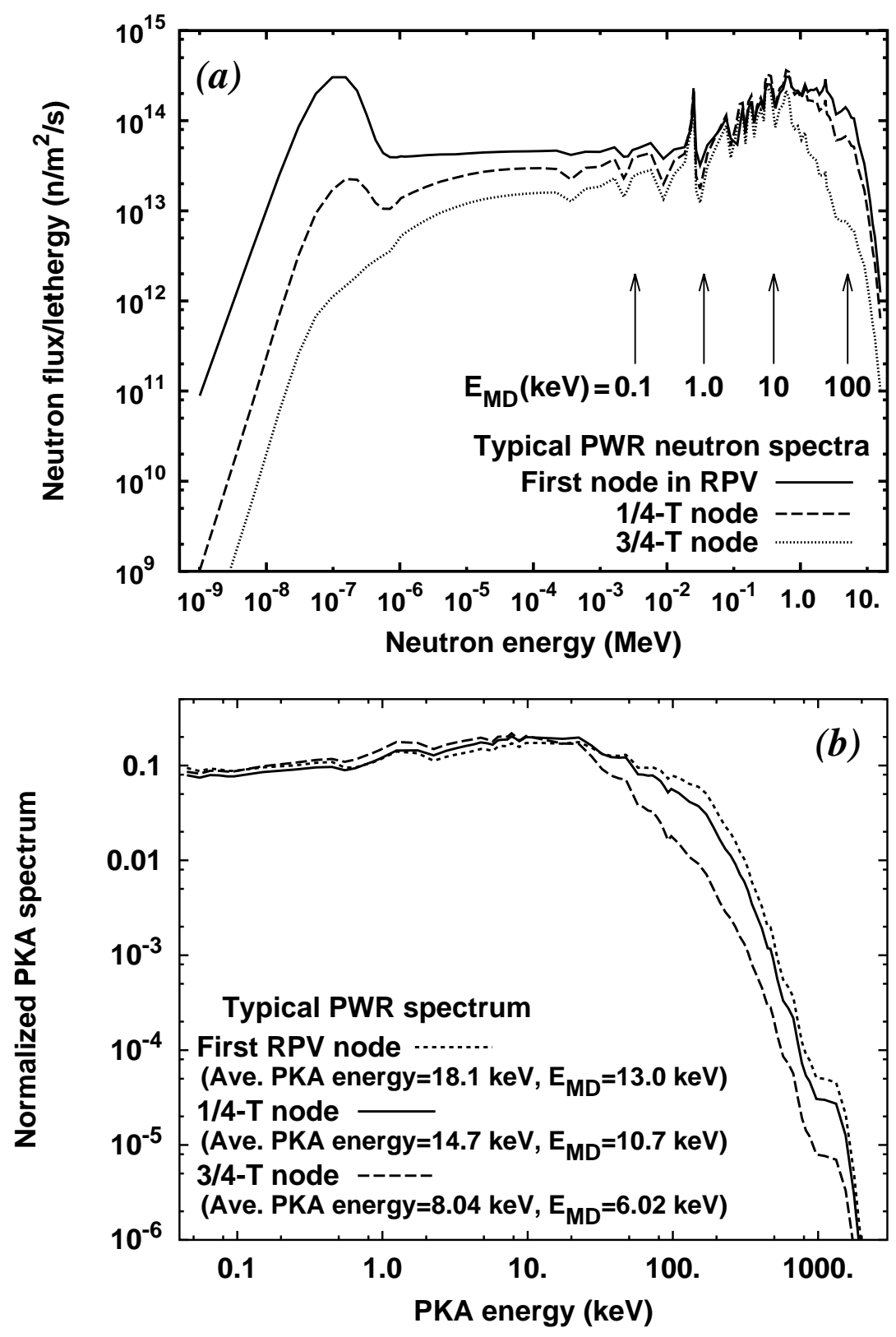

Figure 1 - Neutron energy spectra (a) and PKA spectra (b) for three locations in a typical pressurized water reactor pressure vessel.

1(a) are shown in Fig. 1(b). The average PKA energy and its corresponding damage energy are indicated for each PKA spectrum. The difference between PKA and damage energy is discussed in the next section.

\section{Cascade Simulations}

The MD displacement cascade simulations were carried out using the MOLDY code [14], and the interatomic potential for iron that is described in Refs. [15 and 16]. The MOLDY code simply describes elastic collisions between atoms and does not account for energy loss mechanisms such as electronic excitation and ionization. Therefore, the 
cascade energy $\mathrm{E}_{\mathrm{MD}}$ is analogous to the damage energy in the secondary displacement model by Norgett, Robinson, and Torrens (NRT) [17]. PKA energies that correspond to a given $\mathrm{E}_{\mathrm{MD}}$ can be calculated using the procedure described in Ref. [17], and the neutron energy required to generate PKA with a given average energy can be calculated for an elastic collision. For example, the highest MD cascade energy used in this work is 100 $\mathrm{keV}$. A $100 \mathrm{keV}$ damage energy corresponds to an iron PKA energy of $\sim 176 \mathrm{keV}$, which is the average recoil energy from a collision with a $5.1 \mathrm{MeV}$ neutron. Thus, the simulations encompass most of the neutron energy range experienced by fission reactor components.

The energy dependence of several primary damage parameters have been obtained from the MD simulations. First is the total number of stable displacements created, with vacancies and interstitials being formed in equal numbers. These include all surviving defects present after in-cascade recombination is complete and the simulation cell has returned to thermal equilibrium. A simulation time of about 15 to $20 \mathrm{ps}$ is required to reach this condition at the highest energies. Second, because many of the surviving point defects are contained in small clusters, rather than as isolated defects, the fraction of surviving vacancies and interstitials contained in clusters is determined. It is convenient to express these parameters as a fraction of the displacements predicted by the NRT model [17].

The energy dependence observed in the vacancy and interstitial cluster size distributions suggested that some method of accounting for their spectrum dependence should also be included in this analysis. A single parameter was chosen for both types of defect size distribution for this initial investigation, the number of defects in clusters above a specified size. Clusters containing 5 or more vacancies were observed only at cascade energies of $2 \mathrm{keV}$ and higher, and interstitial clusters of 10 or more were observed only at $20 \mathrm{keV}$ and above. These apparent thresholds made the values of 5 and 10 convenient choices to evaluate the effect of energy on the vacancy and interstitial defect cluster size distributions, respectively. In-cascade clustering is significant because such clusters provide nuclei for the growth of larger defects and their formation directly within the cascade means that extended defects can evolve more quickly than if the clusters were formed only by the much slower process of classical nucleation. The presence of relatively large in-cascade clusters could disproportionately contribute to the nucleation of defects such as interstitial loops and microvoids. The number of vacancies and interstitials in "large" clusters is also expressed as a fraction of the NRT displacements at each energy.

An initial statistical analysis of the cascade database up to $50 \mathrm{keV}$ has been published previously [6], and the energy dependence of total defect survival and interstitial clustering values are discussed in Ref. [18]. Since that time, a series of eight $100 \mathrm{keV}$ simulations have been completed and the vacancy cluster analysis has been carried out on the complete database. The $100 \mathrm{keV}$ results fall on a smooth extrapolation of the lower energy data, as shown in Fig. 2 for the total defect survival fraction. At each energy, the data point is an average of between 8 and 128 cascades, with error bars shown indicating the standard error on the mean. These error bars are almost too small to be observed at the higher energies in Fig. 2, indicating that the minimum observed in the curve near $20 \mathrm{keV}$ is statistically significant. The minimum arises from the extensive subcascade formation 


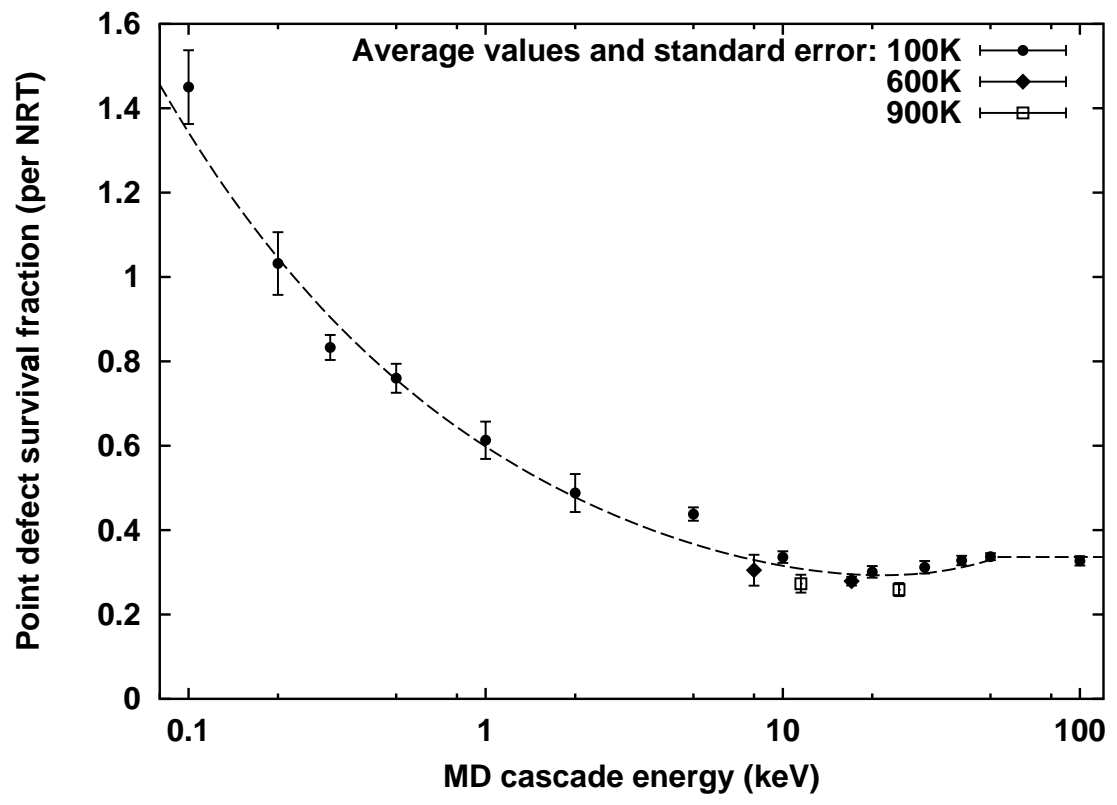

Figure 2 - Energy dependence of total point defect survival obtained from $M D$ cascade simulations.

that occurs above $10 \mathrm{keV}$ [6,8-10]. Although a detailed comparison of the results of similar work is beyond the scope of this paper, it is worth pointing out that similar observations on the energy dependence of defect formation have been made by others who used a different interatomic potential for iron [19].

The interstitial clusters formed in these iron cascade simulations tend to be well defined, with most clusters consisting of nearest neighbor arrangements of $\langle 111\rangle$ or $<110>$ dumbbells, or $<111>$ crowdions. Occasionally, interstitials may be added to a cluster in the second nearest neighbor location or the atomic arrangements can be more complex [20]. The use of computer visualization and animation software enables such interstitials to be clearly identified as part of the cluster. However, the vacancy clusters produced in these iron cascades tend to be diffuse, with clearly correlated arrangements out to the fourth nearest neighbor distance $[9,10]$. As a result, two different criteria were employed in the vacancy cluster analysis to define what constitutes a vacancy cluster. The criterion were that all vacancies in the cluster be within: (1) the second $\left(\mathrm{d}_{2 \mathrm{nn}}=0.2867 \mathrm{~nm}\right)$ or, (2) the fourth $\left(\mathrm{d}_{4 \mathrm{nn}}=0.4754 \mathrm{~nm}\right)$ nearest neighbor distance of another vacancy in the cluster. Application of the fourth nearest neighbor criterion nearly doubles the fraction of vacancies in clusters, but is supported by Monte Carlo aging studies of cascade debris [10, 21].

The total fraction of vacancies in clusters for both the $2 \mathrm{nn}$ and $4 \mathrm{nn}$ clustering criteria is shown in Fig. 3(a) and the fraction of vacancies in clusters of 5 or more using the same criteria is shown in Fig. 3(b). For purposes of comparison, the previously derived trend lines for the interstitial clustering parameters from Ref. [10] are also shown in Figs. 3(a) and (b). At low energies, the dependence of vacancy clustering on cascade energy is noticeably different from that of the interstitials. The fraction of vacancies (per NRT displacement) continues to increase down to the lowest energies, whereas the interstitial 

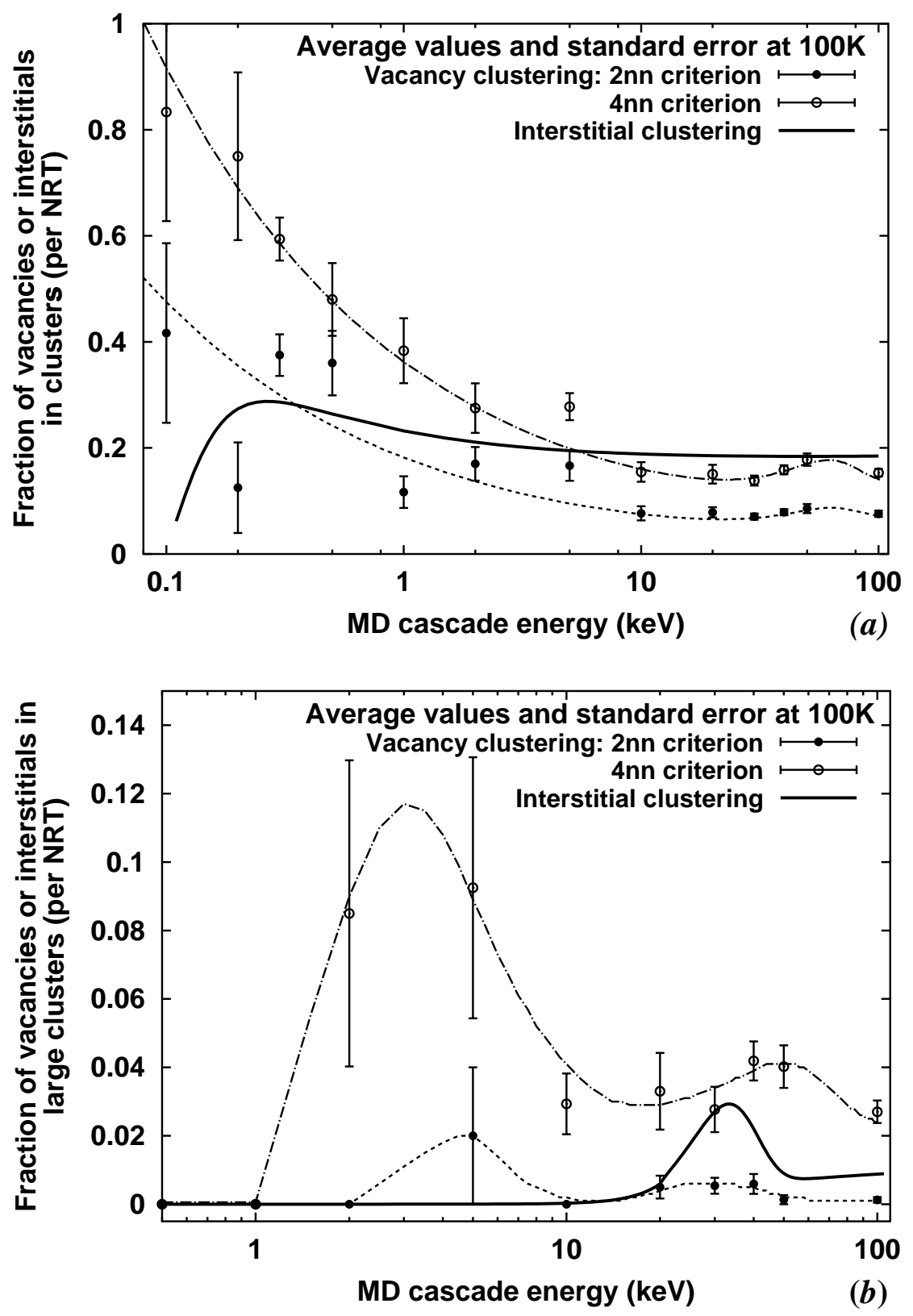

Figure 3 - Energy dependence of normalized primary damage parameters from MD cascade simulations: (a) total vacancies and interstitial in clusters, and (b) vacancies in clusters of 5 or more and interstitials in clusters of 10 or more.

clustering fraction decreases below about $300 \mathrm{eV}$. A much larger fraction of the vacancies than interstitials are in clusters at low energies, and the decrease with energy is much more rapid for the vacancies. At high energies, the clustering fraction for both defect types becomes nearly asymptotic, with some indication of a peak in the vacancy clustering curve near $50 \mathrm{keV}$. A somewhat larger fraction of the interstitials are in clusters at high energies, even for the less restrictive $4 \mathrm{nn}$ vacancy clustering criterion.

The analysis of the cluster size distributions revealed further differences between the clustering behavior of vacancies and interstitials. Fewer large vacancy clusters are 
observed than interstitials, and interstitial clusters containing as many as 33 defects have been observed, but the largest vacancy cluster in the database is 14 . This contributed to the decision to choose a smaller cluster size of 5 as the cutoff for the "large" cluster designation for vacancies whereas 10 was used for the interstitials. As shown in Fig. 3(b), the fraction of interstitials in large clusters appears to peak near $30 \mathrm{keV}[10,18]$. Two peaks are observed for the vacancy cluster parameter near 3 and $40 \mathrm{keV}$. The peaks are small for the $2 \mathrm{nn}$ criterion but well developed for the $4 \mathrm{nn}$ case. No simple explanation has been found for the structure in the curves in Fig. 3(b). The high-energy peaks may reflect a maximum energy density that is associated with the breakup of the cascade into subcascades.

\section{Application of MD Results to Damage Attenuation}

\section{Conventional Estimates of Damage Attenuation Through an RPV}

Prediction of mechanical property changes through the thickness of an RPV are required for routine reactor operations, e.g. pressure-temperature limits for reactor startup, and analysis of accident conditions such pressurized thermal shock. Unfortunately, the available data on damage attenuation are equivocal and in some cases, controversial $[22,23]$. A recent, but very limited data set from the Japan Power Demonstration Reactor (JPDR) is shown in Fig. 4 [24]. Measurements of the Charpy shift at $41 \mathrm{~J}$ are shown for two locations about 18 and $71 \mathrm{~mm}$ deep in the vessel. Only limited inferences can be drawn from this data because: (1) the shift is relatively small due to the low maximum JPDR dose of about $2 \times 10^{22} \mathrm{n} / \mathrm{m}^{2}$, and (2) the total vessel thickness is much less than that of most commercial RPVs. However, the JPDR data are consistent with some interpretations of other data which suggest that mechanical property changes can be

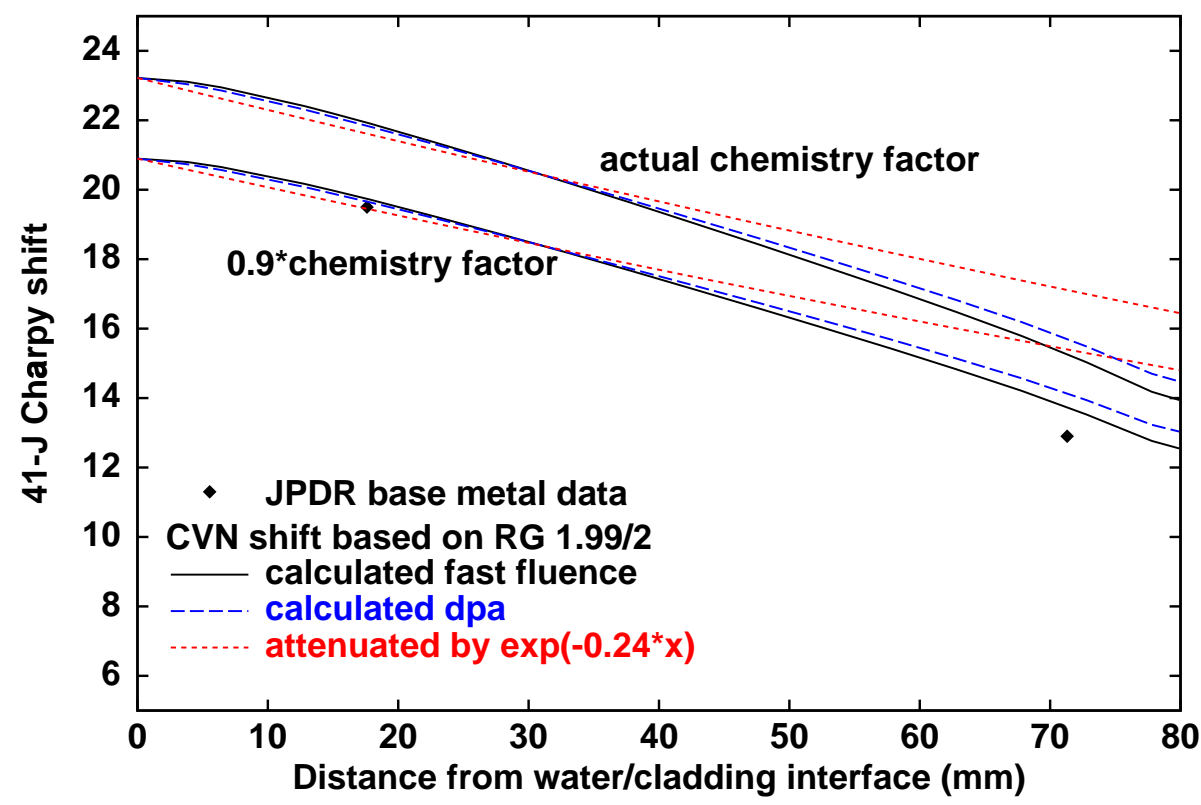

Figure 4 - Comparison of JPDR base metal data and predictions of RG-1.99/2 with various attenuation models. 
attenuated faster than would be predicted based on either dpa or the exponential attenuation formula employed in RG-1.99/2 [22].

This point is illustrated by the two sets of curves in Fig. 4 which compare calculations of the expected attenuation of the Charpy shift through the vessel using the correlation from RG-1.99/2. The neutron fluence needed to compute the shift at each depth was determined in three ways : (a) the actual calculated fast fluence, (2) proportional to the calculated dpa, and (3) proportional to RG-1.99/2's exponential formula. The upper set of curves was obtained using the chemistry factor calculated from the material's measured composition, and the lower curves were obtained by a simple ratio procedure to obtain agreement with the data at $18 \mathrm{~mm}$. In spite of the limited range of the data, the exponential attenuation is clearly very conservative with respect to the data. Of course, the small shift and known scatter in Charpy data [25] mean that this data does not provide a definitive demonstration of faster than dpa attenuation.

A further comparison of different attenuation assumptions is shown in Fig. 5 for the same PWR neutron spectrum discussed above [11]. Figure 5(a) compares the RG-1.99/2 attenuation formula with the actual dpa, neutron fluence with $\mathrm{E}>0.1 \mathrm{MeV}$, and neutron fluence with $\mathrm{E}>1.0 \mathrm{MeV}$. A value for the exposure parameters at the water/RPV interface was obtained by linear interpolation between the values at the last node in the water and the first node in the RPV. The exposure parameters in Fig. 5(a) have been normalized using these interpolated values. For this thicker vessel geometry, deviations between the exponential attenuation formula and dpa are also seen, and the exponential is not conservative at every point. The ratio based on the exponential is nearly the same as fast fluence $(\mathrm{E}>1.0 \mathrm{MeV})$ at the $1 / 4-\mathrm{T}$ position and closer to dpa beyond the 3/4-T position.

The Charpy shifts predicted with this PWR spectrum for a weld with $0.25 \mathrm{wt} \% \mathrm{Cu}$ and $0.75 \mathrm{wt} \% \mathrm{Ni}$ at a fast fluence of $2 \times 10^{23} \mathrm{n} / \mathrm{m}^{2}$ are shown in Fig. 5(b). The procedure of RG1.99/2 was used for each exposure parameter in Fig. 5(a), i.e. the shift was calculated by attenuating the surface fluence with $\mathrm{E}>1.0 \mathrm{MeV}$ according to the attenuation ratios obtained for dpa, fluence with $\mathrm{E}>0.1 \mathrm{MeV}$, or the exponential function. Because of the fluence dependence in the embrittlement correlation, the Charpy shift reduces more slowly through the vessel than does any of the exposure parameters. The relative attenuation in Charpy shift is also a function of the neutron fluence as shown in Table 1, where ratios of the exposure parameters and predicted Charpy shifts are compared at two different fluences. Although the exposure ratios are independent of fluence, the predicted Charpy shift profile becomes flatter as the fluence increases. For this full-thickness PWR RPV, the overall attenuation ratios based on the exponential formula are similar to those based on dpa. The agreement is not as good for a thinner BWR vessel (see for example Figure 4).

\section{Through-Thickness Variation in MD-based Parameters}

Energy dependent functions were obtained by fitting the data in Figs. 2 and 3, and these were used to evaluate the effect of the variation in the neutron energy spectrum through an RPV. These energy-dependent functions have a form similar to those derived previously [26], and they were employed in the SPECOMP code [27] to compute effective production cross sections for point defect survival, total vacancy and interstitial clustering, 

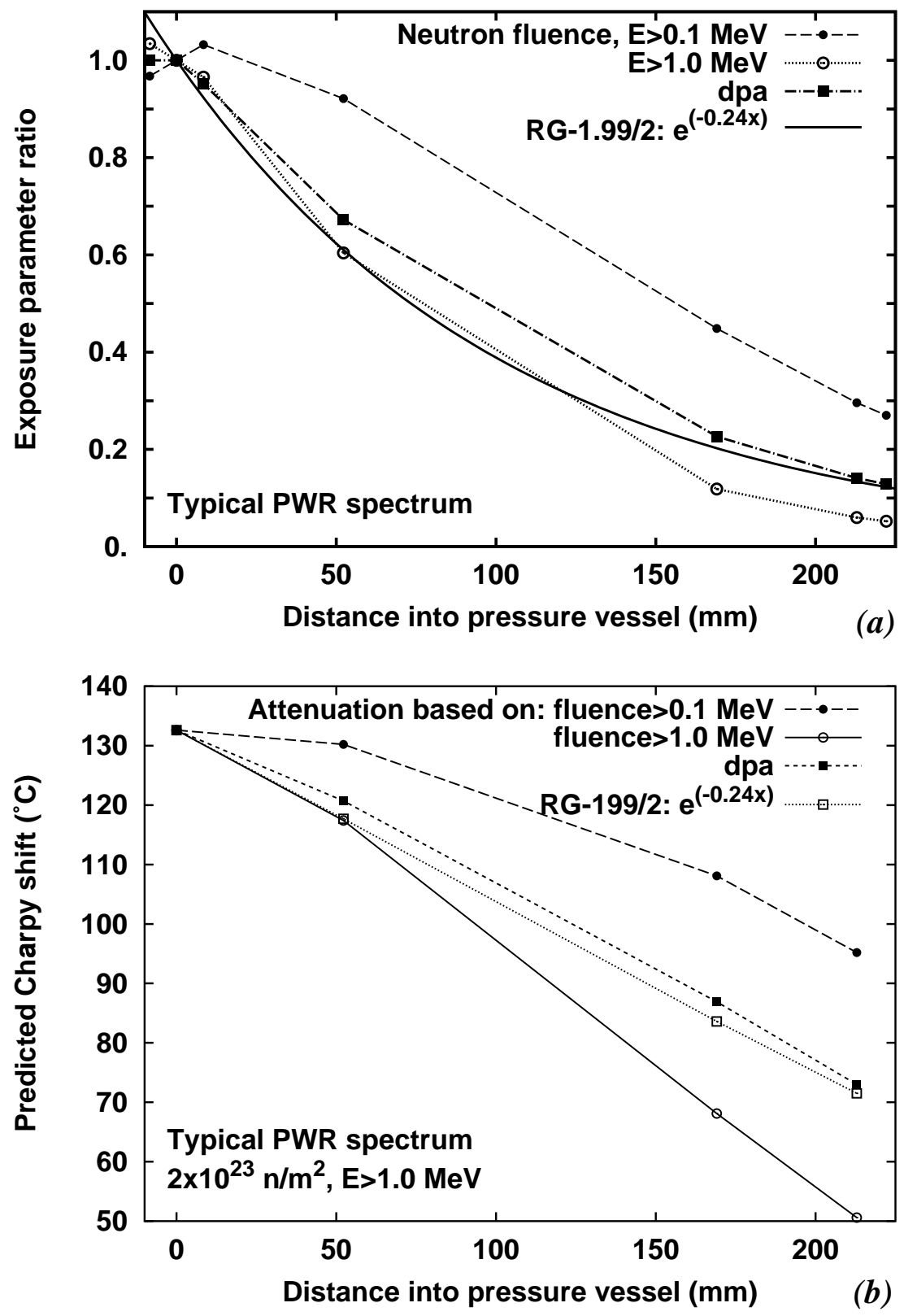

Figure 5 - Comparison of exposure parameter and $41 \mathrm{~J}$ Charpy shift through a typical PWR RPV.

and vacancies and interstitials in large clusters. The values at $50 \mathrm{keV}$ were used for all higher energy cascades. PKA spectra for iron such as those shown in Fig. 1(b) were obtained from SPECTER [13] and used to weight these MD-based cross sections in order to calculate spectrum-averaged values for various locations through the RPV. More details on the SPECOMP/SPECTER calculations can be found in Refs. [12 and 18].

A comparison of the effects of neutron energy spectrum changes through a PWR RPV on the MD-based exposure parameters, fast fluence $(\mathrm{E}>1.0 \mathrm{MeV})$, and dpa is shown in Fig. 6. Total MD defect survival, total interstitials in clusters, and interstitials in large clusters $(\geq 10)$ are shown in Fig. 6(a), and total vacancy clustering and vacancies in large 
Table 1- Attenuation of exposure and damage parameters for typical PWR spectrum

\begin{tabular}{|c|c|c|c|c|c|c|}
\hline & \multicolumn{3}{|c|}{ Ratio: $\frac{\text { Value at outer diameter }}{\text { Value at inner diameter }}$} & \multicolumn{3}{|c|}{ Ratio: $\frac{\text { Value at } 3 / 4-T}{\text { Value at } 1 / 4-\mathrm{T}}$} \\
\hline & \multirow{2}{*}{$\begin{array}{l}\text { Exposure } \\
\text { parameter }\end{array}$} & \multicolumn{2}{|c|}{ 41-J Charpy shift } & \multirow{2}{*}{$\begin{array}{l}\text { Exposure } \\
\text { parameter }\end{array}$} & \multicolumn{2}{|c|}{ 41-J Charpy shift } \\
\hline & & $5 \times 10^{22} \mathrm{n} / \mathrm{m}^{2}$ & $2 \times 10^{23} \mathrm{n} / \mathrm{m}^{2}$ & & $5 \times 10^{22} \mathrm{n} / \mathrm{m}^{2}$ & $2 \times 10^{23} \mathrm{n} / \mathrm{m}^{2}$ \\
\hline $\begin{array}{c}\text { Neutron } \\
\text { fluence, } \\
\mathrm{E}>1.0 \mathrm{MeV}\end{array}$ & 0.0599 & 0.272 & 0.382 & 0.196 & 0.477 & 0.581 \\
\hline $\begin{array}{c}\text { Neutron } \\
\text { fluence, } \\
\mathrm{E}>0.1 \mathrm{MeV}\end{array}$ & 0.296 & 0.619 & 0.718 & 0.487 & 0.761 & 0.830 \\
\hline dpa & 0.144 & 0.434 & 0.551 & 0.337 & 0.631 & 0.720 \\
\hline $\mathrm{e}^{-0.24 \mathrm{x}[\mathrm{in}]}$ & 0.134 & 0.423 & 0.539 & 0.331 & 0.622 & 0.710 \\
\hline
\end{tabular}

( $\geq 5)$ clusters are shown in Fig. 6(b). There is essentially no difference between dpa and either total MD defect survival or total interstitials in clusters in Fig. 6(a). However, a potentially significant difference is seen between dpa and the production of large interstitial clusters. Since these clusters are only produced in relatively high-energy cascades, their production is attenuated more rapidly than dpa. This is consistent with the change in the PKA spectrum through the vessel shown in Fig. 1(b).

Attenuation of the total vacancy clustering fraction in Fig. 6(b) also follows dpa quite closely, whereas the fraction of vacancies in large clusters is attenuated somewhat more slowly than dpa. The vacancy cluster parameters in Fig. 6(b) were derived for the 4nn clustering criterion. Results for the $2 \mathrm{nn}$ criterion are qualitatively similar, with a larger difference between dpa and vacancies in large clusters. The slower-than-dpa attenuation of large vacancy clusters is a result of the lower energy peak shown in Fig. 3(b). The relative importance of vacancy clusters produced by 1 to $10 \mathrm{keV}$ PKA increases as the higher energy PKA are removed from the spectrum.

\section{Summary}

It is now possible to simulate the atomic displacement cascades generated by nearly the complete fission neutron spectrum. The results presented above include cascade energies as high as $100 \mathrm{keV}$, corresponding to a $176 \mathrm{keV}$ PKA, which is the average iron recoil from a neutron with an energy of $5.1 \mathrm{MeV}$. Thus, these results are fully relevant to materials irradiated in any fission reactor environment. Note that the highest average PKA energy obtained from SPECTER for any of the PWR RPV neutron spectra was only 18 $\mathrm{keV}$. This extensive database of cascade simulations can now be used to derive statistically-relevant primary damage parameters and provide a basis for analyzing 

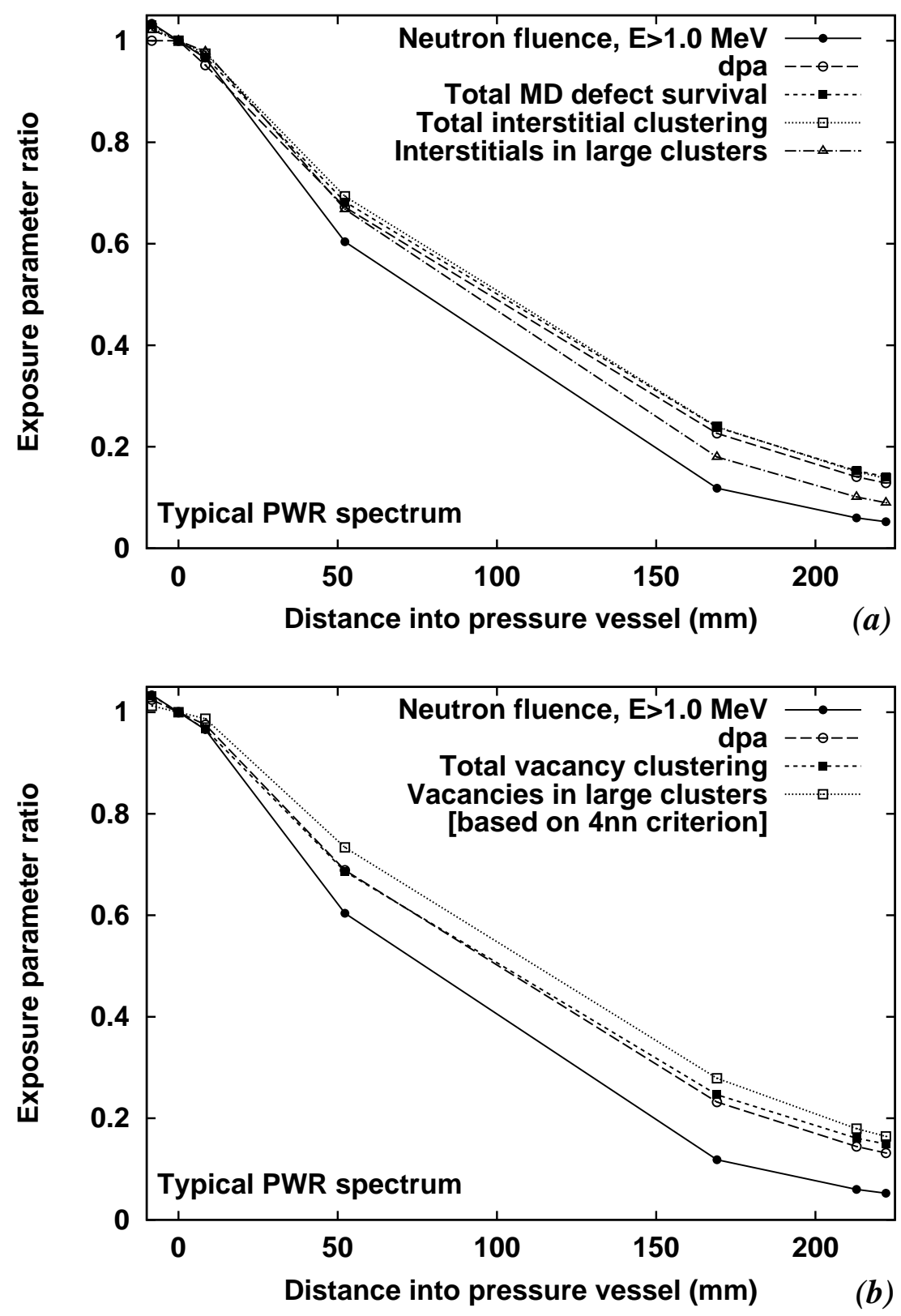

Figure 6 - Attenuation of MD-based exposure parameters compared with fast fluence and dpa.

differences in radiation environments.

Primary damage parameters derived from the MD cascade simulations have been used to obtain effective defect production cross sections for several locations through typical PWR and BWR reactor pressure vessels for comparison with conventional exposure parameters such as dpa and fast fluence. The analysis indicates that the deviations from dpa-like attenuation are rather modest. This implies that neutron energy spectrum changes due to attenuation should be well accounted for through the use of dpa as long as total defect production is the parameter controlling mechanical property changes. Although the results were not presented here, the spectrum-averaged defect production cross section 
derived for PWR and BWR vessels were nearly identical, implying that spectrum differences should not contribute to differences in RPV embrittlement between the two reactor types.

The most significant difference between any of the MD-based parameters and dpa was for the production of large interstitial clusters. If these clusters contribute significantly to embrittlement, attenuation of mechanical property changes could be faster than that predicted by dpa attenuation. This could be offset by the impact of large vacancy clusters since they were attenuated slightly more slowly than dpa. However, it is not possible to predict mechanical property changes simply on the basis of primary damage formation. The spectrum-averaged defect production cross sections will be used to develop an improved description of the radiation damage source terms in a kinetic embrittlement model that has been used to predict radiation-induced mechanical property changes based on microstructural evolution.

Acknowledgments

Research sponsored by the Office of Nuclear Regulatory Research, U.S. Nuclear Regulatory Commission under inter-agency agreement DOE 1886-N695-3W with the U.S. Department of Energy and by the Division of Materials Sciences and Engineering, U.S. Department of Energy under contract DE-AC05-00OR22725 with UT-Battelle, LLC, and the Office of Fusion Energy Sciences, U.S. Department of Energy at the Pacific Northwest National Laboratory.

\section{References}

[1] "Radiation Embrittlement of Reactor Vessel Materials," Regulatory Guide 1.99, Revision 2, U.S. Nuclear Regulatory Commission, May 1988, available from the U.S. National Technical Information Service, Springfield, VA.

[2] Randall, P. N., "Basis for Revision 2 of the U.S. Nuclear Regulatory Commission's Regulatory Guide 1.99," Radiation Embrittlement of Nuclear Reactor Pressure Vessel Steels: An International Review, STP 909, L.E. Steele, Ed., American Society for Testing and Materials, West Conshohocken, PA, 1986, pp. 149-162.

[3] Eason, E. D., Wright, J. E., and Odette, G. R., "Improved Embrittlement Correlations for Reactor Pressure Vessel Steels,” NUREG/CR-6551, MCS 970501, U.S. Nuclear Regulatory Commission, November 1998, available from the U.S. National Technical Information Service, Springfield, VA.

[4] Recommendations of IAEA Specialists Meeting on Radiation Damage Units in Graphite and Ferritic and Austenitic Steels, Nuclear Engineering and Design, 1975, 33, p. 48.

[5] Stoller, R. E. and Odette, G. R., "Recommendations on Damage Exposure Units for Ferritic Steel Embrittlement," Journal of Nuclear Materials 1992, 186, pp. 203205.

[6] Stoller, R. E. and Calder, A. F., "Statistical Analysis of a Library of Molecular Dynamics Cascade Simulations in Iron at 100K, "Journal of Nuclear Materials 2000, 283-287, 186, pp. 203-205. 
[7] Phythian, W. J., Stoller, R.E., Foreman, A. J. E., Calder, A. F., and Bacon, D. J., “A Comparison of Displacement Cascades in Copper and Iron by Molecular Dynamics and Its Application to Microstructural Evolution," Journal of Nuclear Materials 1995, 223, pp. 245-261.

[8] Stoller, R.E., "Point Defect Survival and Clustering Fractions Obtained From Molecular Dynamics Simulations of High Energy Cascades,' Journal of Nuclear Materials 1996, 233-237, pp. 999-1003.

[9] Stoller, R.E., Odette G.R., and Wirth, B. D., "Primary Damage Formation in bcc Iron," Journal of Nuclear Materials 1997, 251, pp. 49-69.

[10] Stoller, R.E., "The Role of Cascade Energy and Temperature in Primary Defect Formation in Iron," Journal of Nuclear Materials 2000, 276, pp. 22-32.

[11] Albert,T.E., Gritzner,M.L., Simmons, G.L., and Straker, E.A., "PWR and BWR Radiation Environments for Radiation Damage Studies, "EPRI NP-152, Electric Power Research Institute, Palo Alto, CA, September 1977.

[12] Stoller, R.E. and Greenwood, L.R., "An Evaluation of Neutron Energy Spectrum Effects Based on Molecular Dynamics Displacement Cascade Simulations," Effects of Radiation on Materials, STP 1366, M. L. Hamilton, A. S. Kumar, S. T. Rosinski, and M. L. Grossbeck, Eds., American Society for Testing and Materials, West Conshohocken, PA, 2000, pp. 548-559.

[13] Greenwood, L.R. and Smither, R.K., "SPECTER: Neutron Damage Calculations for Materials Irradiations," ANL/FPP/TM-197, Argonne National Laboratory, Argonne, IL, January 1985.

[14] Finnis, M.W., "MOLDY6-A Molecular Dynamics Program for Simulation of Pure Metals,” AERE R-13182, UKAEA Harwell Laboratory, Harwell, UK, 1988.

[15] Finnis, M.W. and Sinclair, J.E., "A Simple Empirical N-body Potential for Transition Metals," Philosophical Magazine-A 1984, 50, pp. 45-55 and Erratum, Philosophical Magazine-A, 1986, p. 161.

[16] Calder, A.F. and Bacon, D.J., "A Molecular Dynamics Study of Displacement Cascades in $\alpha$-iron," Journal of Nuclear Materials 1993, 207, pp. 25-45.

[17] Norgett, M.J., Robinson, M.T., and Torrens, I.M., “A Proposed Method of Calculating Displacement Dose Rates," Nuclear Engineering and Design 1975, 33, pp. 50-54.

[18] Stoller, R.E. and Greenwood, L.R., "A Comparison of the NRT Displacement Model and Primary Damage Formation Observed in Molecular Dynamics Cascade Simulations," presented at the 10th International Symposium on Reactor Dosimetry, 12-17 September 1999, Osaka, Japan, to be published by the American Society of Testing and Materials in ASTM STP 1398

[19] N. Soneda and T. Diaz de la Rubia, "Defect Production, Annealing Kinetics, and Damage Evolution in $\alpha$-Fe: An Atomic-scale Computer Simulation," Philosophical Magazine A 1998, 78, pp. 995-1019.

[20] Stoller, R.E., "Molecular Dynamics Simulations of High Energy Cascades in Iron," Microstructure of Irradiated Materials, I. M. Robertson, L. E. Rehn, S. J. Zinkle, and W. J. Phythian, Eds., Materials Research Society, Pittsburgh, PA, 1995, pp. 2126. 
[21] B. D. Wirth and G. R. Odette, "Kinetic Lattice Monte Carlo Simulations of Cascade Aging in Iron and Dilute Iron-Copper Alloys," Multiscale Modeling of Materials, V. V. Butalov, T. Diaz de la Rubia, P. Phillips, E. Kaxiras, and N. Ghoniem, Eds., Materials Research Society, Pittsburgh, PA, 1999, pp. 211-216.

[22] Gold, R. and McElroy, W. N., "Radiation-Induced Embrittlement in Light Water Reactor Pressure Vessels," Nuclear Engineering and Design 1987, 104, pp. 155 174.

[23] Kussmaul, K., Föhl, J., and Weissenberg, T., "Assurance of the Pressure Vessel Integrity with Respect to Irradiation Embrittlement: Activities in the Federal Republic of Germany," Radiation Embrittlement of Nuclear Reactor Pressure Vessel Steels, ASTM STP 1011, L. E. Steele, Ed., American Society of Testing and Materials, West Conshohocken, PA, 1989, pp. 3-26.

[24] Suzuki, M., "Investigation of In-service Irradiated pressure Vessel Steel from the Decommissioned Reactor, JPDR,' ICONE-7411, 7th International Conference on Nuclear Engineering, Tokyo, Japan, April, 1999, pp. 1-8.

[25] Nanstad, R. K., McCabe, D. E., and Swain, R. L., "Evaluation of Variability in Material Properties and Chemical Composition for Midland Reactor Weld WF70," Effects of Radiation on Materials, STP 1325, R. K. Nanstad, M. L. Hamilton, F. A. Garner, and A. S. Kumar, Eds., American Society for Testing and Materials, West Conshohocken, PA, 1999, pp. 125-156.

[26] Stoller, R. E. and Greenwood, L. R., "Subcascade Formation in Displacement Cascade Simulations; Implications for Fusion Reactor Materials," Journal of Nuclear Materials 1999, 271\&272, pp. 57-62.

[27] Greenwood, L. R., "SPECOMP Calculations of Radiation Damage in Compounds," Reactor Dosimetry: Methods, Applications, and Standardization, ASTM STP 1001, H. Farrar IV and E. P. Lippincott, Eds., American Society of Testing and Materials, West Conshohocken, PA, 1989, pp. 598-602. 